\title{
Effect of National Early Warning Scoring System Implementation on Cardiopulmonary Arrest, Unplanned ICU Admission, Emergency Surgery, and Acute Kidney Injury in an Emergency Hospital, Egypt
}

\section{Mohamed Naeem Badr (iD) Nahla Shaaban Khalil' Ahmed Mohamed Mukhtar ${ }^{2}$ \\ 'Critical Care and Emergency Nursing, Faculty of Nursing, Cairo University, Cairo, Egypt; ${ }^{2}$ Anesthesia and Critical Care, Faculty of Medicine, Cairo University, Cairo, Egypt}

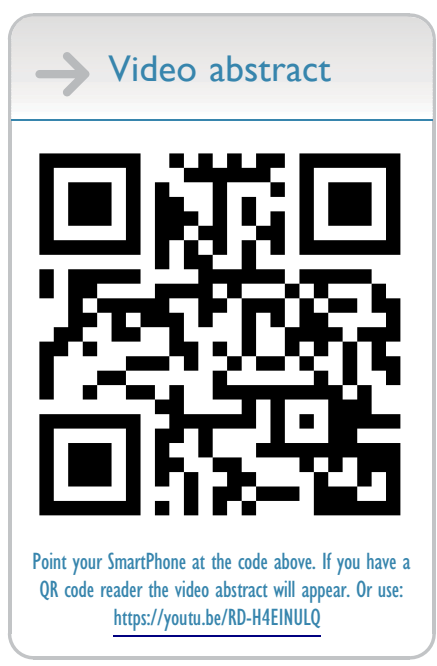

Correspondence: Mohamed Naeem Badr Tel +20 100990 I4l4

Email mohamed.badr2002@gmail.com
Purpose: To evaluate the effect of national early warning scoring system (NEWS) implementation in identifying patients at risk of clinical deterioration at an emergency hospital. Background: Early warning score has been developed to facilitate early detection of deterioration by categorizing a patients' severity of illness and prompting nursing staff to request a medical review at specific trigger points.

Patients and Methods: A prospective, control/intervention groups', quasi-experimental design was utilized. A sample of 364 adult patients were admitted to the inpatient unit at an emergency hospital for six months. The patients were divided into a study group (174 patients) and a control group (190 patients). All study patients were followed up to either death or hospital discharge before and after implementing a new observation chart. The patients' outcomes were compared and analyzed between both groups.

Results: In the intervention period, compared to the control period, a significant reduction was seen in the number of cardiopulmonary arrest $(4.7 \% \mathrm{vs} 1.1 \%, \mathrm{p}=0.046)$, unplanned ICU admission $(5.3 \%$ vs $1.7 \%, p=0.049)$, emergency surgery $(6.3 \%$ vs $0 \%, p=0.001)$, acute kidney injury $(6.8 \%$ vs $1.1 \%, \mathrm{p}=0.006)$. As well, there was a significant increase in the number of patients receiving medical reviews following clinical deterioration in terms of escalation plan $(3.2 \%$ vs $26.4 \%, \mathrm{p}=<0.001)$.

Conclusion: The implementation of NEWS was associated with a significant improvement in patients' outcomes in hospital wards, increases in the frequency of vital signs measurements, and an increase in the number of medical reviews following clinical instability.

Keywords: early warning score, NEWS, patients' outcomes, clinical deterioration

\section{Introduction}

Failure in recognizing that a patient's condition is deteriorating in the hospital setting, a state often characterized by significant physiological abnormalities for over 24 hours, ${ }^{1-3}$ and manifested clinically as derangements in vital signs, ${ }^{4}$ leads to delays in appropriate management and delayed detection of deterioration. ${ }^{5}$ Such worsening physiology is associated with serious adverse events that lead to lifethreatening conditions, prolongation of hospitalization, and significant disability or 
incapacity. These delays are associated with unplanned admissions or readmissions into the intensive care unit (ICU), cardiac arrests, and unexpected deaths. ${ }^{6}$

Early detection of clinically unstable patients is the cornerstone in improving the patient' outcomes that require a series of steps, including vital signs documentation and interpretation, subsequent meaningful communication, timely and appropriate management from the medical emergency team (MET). ${ }^{7}$ Along these lines, there was a need to utilize "track and trigger" (T\&T) systems in which vitalsigns data are collected periodically from patients and then scored according to their abnormality. ${ }^{8}$ One of these systems is the National Early Warning Score. ${ }^{9}$

In 2012, an attempt was made in the United Kingdom to improve the assessment and documentation of vital signs in hospitals by introducing a unified, standardized concept on a national basis. This concept was called the National Early Warning Score (NEWS), ${ }^{10}$ and many hospitals are now using early warning scoring systems as objective methods to identify patient deterioration and ensure timely care. It also provides a standardized observation system and continuity of patient care between wards ${ }^{8}$ when these early warning systems are used in conjunction with an established MET, those patients flagged for particular attention and can be seen early by a specialized team, reducing the potential for adverse events. ${ }^{6}$

Further, in accordance with the recommendation of the World Health Organization (WHO) and the Royal College of Physicians of London (RCP), by using national early warning score during the pandemic outbreak ${ }^{11,12}$ NEWS2 appeared to be a strong predictor of intra-hospital mortality for COVID-19 patients. This is of enormous importance since it supports the fact that NEWS2 can support clinical judgment and provide a standardized communication tool that could be practically feasible in a short time scale and in the context of strained resources and operational pressure faced by hospitals during the emergency phase of the COVID-19 pandemic outbreak. ${ }^{13}$ As it will ensure that patients who are deteriorating, or at risk of deteriorating, will have a timely initial assessment by a competent clinical decision maker. ${ }^{12}$

Our study aimed to assess the effect of national early warning scoring system (NEWS) implementation in identifying patients at risk of clinical deterioration at an acute emergency ward. On the other hand, a large perspective study conducted at an acute medical ward, demonstrated NEWS is a valuable tool for the patients' risk stratification and adverse events prediction. ${ }^{14}$
We hypothesized that Nurses' implementation of the early warning scoring system would improve the patients' outcomes in terms of a decrease in the incidence of cardiopulmonary arrest, decrease in the incidence of unplanned ICU admission, decrease in the incidence of emergency surgery, decrease in the incidence of acute kidney injury and increase the frequency of a medical review following clinical deterioration.

In this research, we used a theoretical framework that enables the nurses to identify patients at risk of deterioration and request a medical review on time. In addition to providing a methodology and a standard approach for communicating the clinical instability. ${ }^{15}$

\section{Patients and Methods Design}

A prospective control/intervention groups' quasiexperimental research design was conducted for all adult patients admitted to the selected inpatient unit.

\section{Study Setting and Sample}

This study was conducted at an inpatient unit in an emergency hospital. It provides specialized medical and surgical care to seriously ill patients. It encompassed different surgical specialties, such as general surgery, cardiothoracic, vascular, urology, hepatobiliary, and orthopedic, as well as medical specialties, such as gastroenterology, renal, and hepatology. The nursing staffing level in the general ward was 3 patients per nurse.

A sample of 364 adult male and female patients that met the inclusion criteria were divided into a study group (174 patients) and a control group (190 patients). The criteria for inclusion included all adult patients admitted to the studied unit. The exclusion criteria were patients, who were less than 18 years of age, were pregnant, and patients readmitted to the unit during the study. These criteria were in accordance with the guidelines of the Royal College of Physicians, 2017. ${ }^{9}$ We selected the preceding event when the patient had experienced multiple events.

All adult patients admitted to a selected unit and who met the inclusion criteria were included during the study periods. In addition, a simple random sample was chosen according to the following equation with a significance level $95 \%$ and a margin of error 0.05 :

$$
n=\frac{z^{2} * p *(1-p) / e^{2}}{1+\frac{z^{2} * p *(1-p)}{e^{2} * N}}
$$


Where $\mathrm{z}$ is the $\mathrm{z}$-score associated with the significance level chosen, $\mathrm{P}$ is the percent in a population estimated to be 0.5 , e is the margin of error, $\mathrm{N}$ is the population size. The calculated sample according to the equation was $\mathrm{n}=300$.

The actual sample size was then taken to be more than 300 to allow for the dropout of some patients so that the final sample size would not be less than 300 . The actual sample size was 364 , after excluding patients who were less than 18 years of age, were pregnant, patients readmitted to the unit during the study, and patients who refused to participate. Then, they are randomly assigned to two groups of patients, study and control (Figure 1).

Data were collected for the control group from July to September 2018; then, the educational program was implemented for two months from October to November 2018, and finally, the study group from December 2018 to February 2019. There were no changes between the two periods regarding (technologies and staff ratio).

The study protocol was approved by the Ethics Committee of the Faculty of Nursing, Cairo University, Egypt, according to the Institutional Review Board for the Protection of Human Rights with reference number IORG 0003381-IRB 00004025-FWA 00026458.

All patients included in the study were informed about its purpose, procedure, benefits, and nature. They were ensured that participation in this study was voluntary, and confidentiality and anonymity of each patient were assured through coding all data. They had the right to withdraw from the study at any time without any rationale. Then, written consent was obtained from them.

\section{Instruments}

\section{Instrument I}

Patient's demographic and medical data sheet: It covered data related to age, sex, admission diagnosis, past medical history, patient response to treatment, length of stay, and admission date. Data were obtained from the medical record.

\section{Instrument 2}

National Early Warning Score (NEWS) is a "track and trigger" scale. It is a multi-parameter aggregate scoring system. Measures of respiratory rate, oxygen saturation, body temperature, systolic blood pressure, heart rate, and level of consciousness are rated from 0 to 3 , correlating with their divergence from the expected normal values. The level of consciousness is assessed by the AVPU concept as follows: $(\mathrm{A}=$ alert, $\mathrm{V}=$ verbal stimuli response, $\mathrm{P}=$ pain stimuli response, $\mathrm{U}=$ unresponsive). Any alteration in the level of consciousness gets three points. A score is attributed to each of these parameters, getting one score per parameter, and the scores are summed, and eventual supplemental oxygen increases the score by two points (Table 1 ). ${ }^{10}$

The sum of points is then related to the level of clinical risk for the patient: as follows: low-risk score (0 to 4) so the nurse will reassess the NEWS parameters after 6 hours; medium-risk score (more than 5 or three points in one individual parameter), so that the nurse will reassess after 1 hour and recheck with another nurse; and high-risk score (7 or more); then, the NEWS escalation protocol was activated when the nurse calls the responsible physician.

The escalation protocol serves as clinical decision support for the healthcare staff. The decision support be made up of recommendations on levels of expertise required for the situation, assessment interval, and level of care, and activation of the medical emergency team (MET). ${ }^{10}$

\section{Instrument 3}

An observational checklist: It covered data related to the patients' outcomes, which divided into primary outcome (Cardiopulmonary arrest) and secondary outcomes, which included (unplanned ICU admission, emergency surgery, acute kidney injury based on akin tool adopted by Acute Kidney Injury Network ${ }^{16}$ and incidence of medical reviews following clinical deterioration). It was carried out for each patient in the study and control group. These checklists are used daily before and after utilizing the NEWS until the patient's discharge/death for detecting any occurred outcome.

A standardized instrument (NEWS) was used to collect data, and the developed instruments (observational checklists) were tested for reliability utilizing Inter rater-reliability with Krippendorff's alpha. The reliability values for developed instruments were $0.84,0.91,0.77,0.81$ and 0.79 .

\section{Study Variables}

The SAEs that represent patient's outcomes were as follows: (1) Cardiopulmonary arrest, (2) unexpected death, (3) Unplanned ICU admission, (4) Emergency surgery and (5) acute kidney injury (AKI). These events are all linked to a high mortality rate. However, they additionally have something else in common: all of them relate to previously deviating vital signs. ${ }^{17,18}$ This feature is essential for detecting and intervening these SAEs. 


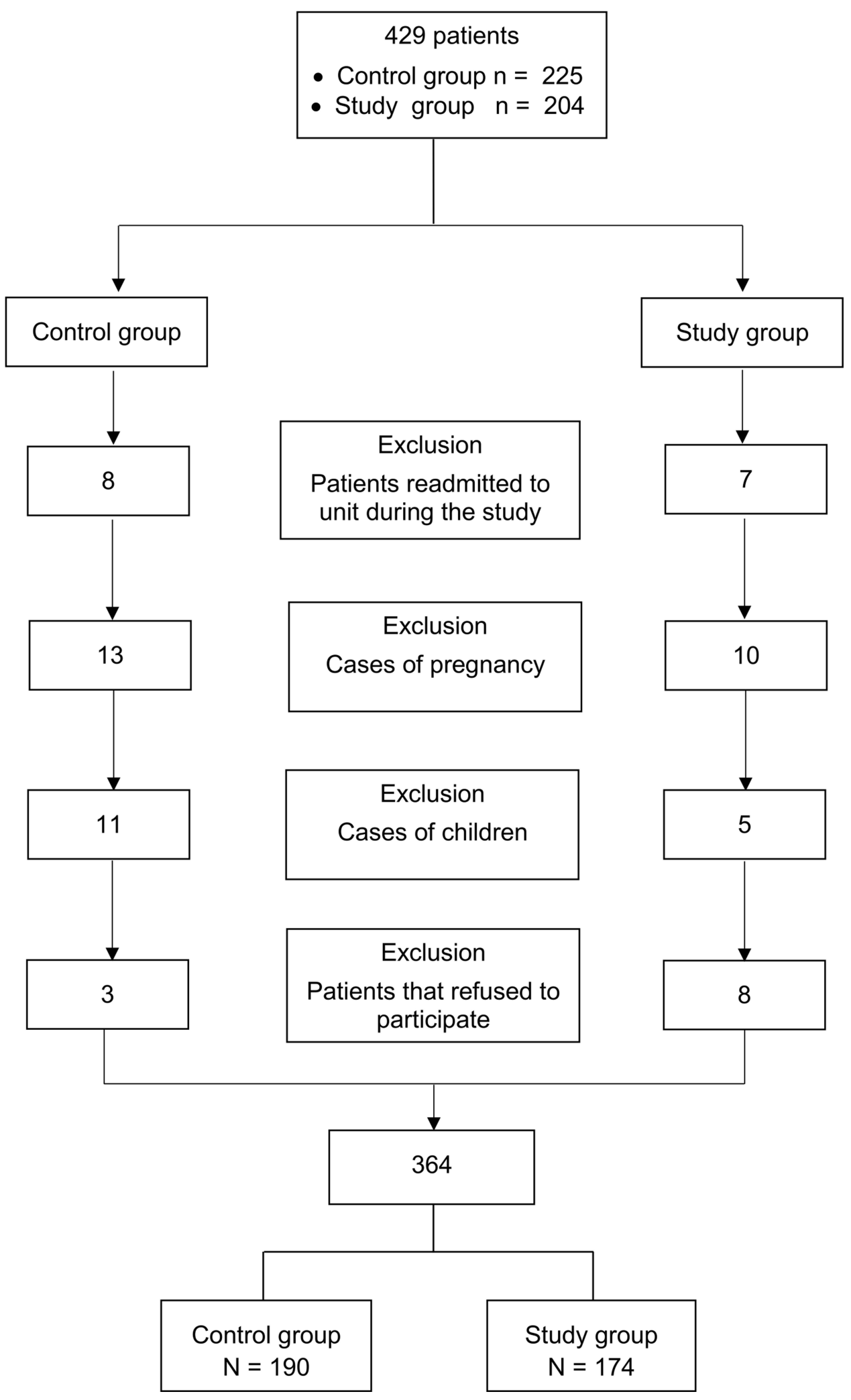

Figure I Flow diagram of patients included.

Note: The actual sample size was 364 , after excluding patients who were less than 18 years of age, were pregnant, patients readmitted to the unit during the study, and patients who refused to participate. 
Table I National Early Warning Score

\begin{tabular}{|c|c|c|c|c|c|c|c|}
\hline Physiological Parameters & 3 & 2 & I & 0 & I & 2 & 3 \\
\hline Respiratory rate & $\leq 8$ & & $9-11$ & $12-20$ & & $21-24$ & $\geq 25$ \\
\hline Oxygen saturation & $\leq 91$ & $92-93$ & $94-95$ & $\geq 96$ & & & \\
\hline Oxygen supplement & & Yes & & No & & & \\
\hline Temperature & $\leq 35.0$ & & $35.1-36.0$ & $36.1-38.0$ & $38.1-39.0$ & $\geq 39.1$ & \\
\hline Systolic blood pressure & $\leq 90$ & $91-100$ & $101-110$ & $111-219$ & & & $\geq 220$ \\
\hline Heart rate & $\leq 40$ & & $4 I-50$ & $51-90$ & $91-110$ & $111-130$ & $\geq|3|$ \\
\hline Level of consciousness & & & & A & & & $\mathrm{V}, \mathrm{P}$ or $\mathrm{U}$ \\
\hline
\end{tabular}

Notes: Adopted from Royal College of Physicians. National Early Warning Score (NEWS): standardizing the assessment of acute illness severity in the NHS. Report of a working party. RCP; $2012^{10}$ and $2017 .^{9}$

Abbreviations: A, Alert; $V$, verbal; $P$, pain; $U$, unresponsive.

The SAEs were defined as follows: Cardiopulmonary arrest was defined as an event in which respiratory and/or cardiopulmonary activity was absent for which the cardiac arrest team initiated cardiopulmonary resuscitation, including chemical, fluid, or mechanical resuscitation; Unexpected death was defined as death without the presence of any form of a "Do Not Attempt Resuscitation" (DNAR) order; Unplanned ICU admission was defined as an admission to the ICU that did not come from the operating theatres, another ICU, or the emergency department. ${ }^{19}$ Emergency surgery was defined as requiring surgery within one hour after the establishment of this decision. ${ }^{18}$

Acute kidney injury (AKI) was added as another important and potentially avoidable AE. It is a syndrome characterized by a sudden loss of renal function resulting in disruption to fluid, acid-base, and electrolyte homeostasis; it is both a frequent cause and consequence of acute illness. ${ }^{20}$ AKI was defined and classified using the AKIN criteria. ${ }^{16}$ AKI stages are determined by the maximum change in either serum creatinine or urine output. It requires at least two serum creatinine values obtained within a period of $48 \mathrm{~h}$ to classify acute kidney injury. ${ }^{21,22}$

\section{Data Collection}

\section{Pre-Intervention}

Data were collected from July 2018 to February 2019. The trained nurses filled out Patients' Socio-demographic, medical datasheet, and observational checklists that monitored the patients' outcomes for the control group within three months. The vital signs of the control group subjects (190) were measured according to the hospital policy.

\section{Education Program}

The researcher trained the emergency nurses on practicing the national early warning scoring system for two months utilizing; lecture, group discussion, and clinical scenarios. The program is designed to help understand vital signs' physiological parameters, reasons for measurement and abnormalities, and establish a communication framework between the health care members. It covered the following learning topics: benefits of NEWS, Six Physiological Parameters included, Outline how NEWS works, threshold and triggers, and demonstrating correct use of NEWS and its clinical response.

\section{Post-Intervention}

The national early warning score was implemented by trained nurses as a new ward observation chart for three months in the setting for the study group subjects (174) as well as the patients' Socio-demographic, medical datasheet, and observational checklist included patients' outcomes were completed again. All study patients were followed up with either death or hospital discharge. During follow-up, data on the length of hospital stay and incidence of requesting a medical review by staff nurses following clinical deterioration were obtained.

The patients' outcomes were compared and analyzed by the researcher between the study and the control group.

\section{Data Analysis}

Upon completing data collection, data were tabulated and analyzed using a statistical package for social sciences (SPSS) program version 25. Relevant statistical analysis was done to test the obtained data. Means and standard 
deviations were used to describe quantitative variables, while independent samples $t$-test was used to test homogeneity between the two groups. A Chi-square test of proportions was used to test the homogeneity of gender between the two groups. The Chi-square test of independence was used to study the difference between the two groups regarding patient outcomes and NEWS escalation categories.

Post hoc tests for the Chi-square test were used to determine categories that are significantly different. Independent samples $t$-test was used to test the difference between the mean frequency of vital signs in both groups. The level of significance was considered at the $5 \%$ level $(\mathrm{P}=0.05)$.

Logistic regression analysis was used to study the effect of the intervention on different outcomes and also to study the effect of gender and age on outcomes to make sure differences in initial age and gender between groups do not affect outcomes. Independent variables were intervention, age, and gender, while the dependent variable was taken as one outcome for every single regression.

\section{Results}

Demographic and Medical Relevant Data

Table 2 During the control period (190) patients were studied compared with (174) patients during the intervention period. The demographics were similar in both two periods. The two-thirds of the control group were males $(61.5 \%)$ (117) compared to $(73 \%)$ (127) of the study group, respectively. Concerning age, it showed that the mean age of the control group was $(40 \pm 17)$. In comparison, the study group was (43 \pm 19$)$. Regarding the length of stay showed that the control group's overall mean days

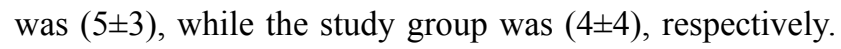
Also, almost two-thirds of both groups had no past medical history. Further, $26.4 \%$ of the control group had diabetes compared to $17.9 \%$ of the study group. In comparison, $21.6 \%$ of the control group had hypertension compared to $15.5 \%$ of the study group.

It is apparent from Table 3 that more than three-fourths of patients were admitted for general surgery in both control and study groups, respectively (83.9\% vs $77 \%$ ). Moreover, more than two-thirds of the control group had gastrointestinal disorders when compared to the study group.
Table 2 Frequency Distribution of the Patients' Demographic Data in Both Study and Control Groups

\begin{tabular}{|c|c|c|}
\hline \multirow[t]{3}{*}{ Sample Variables } & \multicolumn{2}{|c|}{ Study Sample $n=364$} \\
\hline & Control Group & Study Group \\
\hline & No (\%) & No (\%) \\
\hline Number of patients & 190 & 174 \\
\hline \multicolumn{3}{|l|}{ Age } \\
\hline Mean \pm SD & $40 \pm 17$ & $43 \pm 19$ \\
\hline $\mathrm{p}$-value & \multicolumn{2}{|c|}{$P=0.05$} \\
\hline \multicolumn{3}{|l|}{ Gender } \\
\hline Male & 117 (61.5\%) & I 27 (73\%) \\
\hline Female & 73 (38.5\%) & 47 (27\%) \\
\hline $\mathrm{p}$-value & \multicolumn{2}{|c|}{$\mathrm{P}=0.02$} \\
\hline \multicolumn{3}{|l|}{ Length of stay } \\
\hline Mean \pm SD & $5 \pm 3$ & $4 \pm 4$ \\
\hline p-value & \multicolumn{2}{|c|}{$P=0.07$} \\
\hline Past medical history & & \\
\hline No past medical history & $116(61.1 \%)$ & 124 (7I.3\%) \\
\hline \multicolumn{3}{|l|}{ Diabetes } \\
\hline Yes & $50(26.4 \%)$ & 31 (17.9\%) \\
\hline No & I 40 (73.6\%) & I 43 (82.1\%) \\
\hline \multicolumn{3}{|l|}{ Hypertension } \\
\hline Yes & $4 \mathrm{I}(2 \mathrm{I} .6 \%)$ & 27 (I5.5\%) \\
\hline No & 149 (78.4\%) & I 47 (84.5\%) \\
\hline \multicolumn{3}{|l|}{ Cardiac } \\
\hline Yes & $3(1.6 \%)$ & $3(1.8 \%)$ \\
\hline No & 187 (98.4\%) & I7I (98.2\%) \\
\hline Other & $5(2.6 \%)$ & $2(1.2 \%)$ \\
\hline$p$-value & \multicolumn{2}{|c|}{$P=0.24$} \\
\hline
\end{tabular}

Note: Statistically significant at $\mathrm{p}$-value $<0.05$.

\section{Patient's Outcomes}

As can be seen from Table 4, it showed that during the intervention period; there was a significant reduction in the number of cardiopulmonary arrest (9/190 [4.7\%] vs 2/174 $[1.1 \%], \mathrm{p}=0.046)$, a significant reduction in the number of unplanned ICU admission (10/190 [5.3\%] vs 3/174 $[1.7 \%], \mathrm{p}=0.049)$, a significant reduction in the number of emergency surgery $(12 / 190[6.3 \%]$ vs $0 / 174$ [0\%], p = 0.001 ), a significant reduction in the number of total acute kidney injury (13/190 [6.8\%] vs 2/174 [1.1\%], $\mathrm{p}=0.006)$, there was also a significant increase in the number of patients receiving a medical review by physicians as a result of clinical deterioration (Escalation plan) $(6 / 190$ 
Table 3 Frequency Distribution of the Patients' Medical Data in Both Study and Control Groups

\begin{tabular}{|l|c|c|c|}
\hline \multirow{2}{*}{ Number of patients } & All Patients Control Period N (\%) & All Patients Intervention Period N (\%) & P-value \\
\cline { 2 - 4 } & 190 & 174 & \\
\hline Type of admission & $149(78.4 \%)$ & $139(80 \%)$ & $35(20 \%)$ \\
Surgical admission & $41(21.6 \%)$ & & 0.73 \\
Medical admission & & $107(77 \%)$ & $17(12.2 \%)$ \\
\hline Surgical & $125(83.9 \%)$ & $4(2.9 \%)$ & 0.13 \\
General & $8(5.4 \%)$ & $9(6.5 \%)$ & $1(0.7 \%)$ \\
Cardiothoracic & $9(6 \%)$ & $1(0.7 \%)$ & \\
Vascular & $4(2.7 \%)$ & $16(45.7 \%)$ & \\
Urology & $1(0.7 \%)$ & $9(25.7 \%)$ & 0.19 \\
Hepatobiliary & $2(1.3 \%)$ & $10(28.6 \%)$ & \\
Orthopedic & & & \\
\hline Medical & $28(68.3 \%)$ & & \\
Gastrointestinal & $5(19.5 \%)$ & & \\
Renal & $5(12.2 \%)$ & & \\
Hepatic & & & \\
\hline
\end{tabular}

Note: Statistically significant at $\mathrm{p}$-value $<0.05$.

Table 4 Frequency Distribution of the Patients' Outcomes in Both Study and Control Groups

\begin{tabular}{|l|c|c|c|c|}
\hline Outcomes & Control Group & Study Group & Crude OR (95\%) & p-value \\
\hline Total number of patients & 190 & 174 & & \\
Cardio Pulmonary Arrest & $9(4.7 \%)$ & $2(1.1 \%)$ & $0.23(0.00,0.98)$ & 0.046 \\
Unplanned ICU Admission & $10(5.3 \%)$ & $3(1.7 \%)$ & $0.32(0.07,1.3)$ & 0.049 \\
Emergency Surgery & $12(6.3 \%)$ & $0(0 \%)$ & $0(0,0.4)$ & 0.001 \\
Unexpected Death & $7(3.7 \%)$ & $2(1.1 \%)$ & $0.29(0.08,1.1)$ & 0.120 \\
Total Acute kidney Injury & $13(6.8 \%)$ & $2(1.1 \%)$ & $0.16(0.03,0.65)$ & 0.006 \\
Escalation Plan & $6(3.2 \%)$ & $46(26.4 \%)$ & $8.2(2.0,33.0)$ & $<0.001$ \\
\hline
\end{tabular}

Note: Statistically significant at $\mathrm{p}$-value $<0.05$.

[3.2\%] vs 46/174 [26.4\%], p $=<0.001)$, but no other significant statistics in patient-related outcomes. Finally, the OR for all outcomes indicates that the intervention decreases the chance of complications like cardiopulmonary arrest compared to the control group.

\section{Vital Signs Measurements}

It is apparent from Table 5 that reveals that there were highly significant statistical differences between the control and study groups in relation to the frequency of all the vital signs measurement among the studied patient' sample.

\section{Escalation Plan Following Medical Reviews}

It is apparent from Table 6 that reveals that there was a highly significant statistical difference existed between the control and study group in relation to escalation categories based on medical reviews of the health care team among the studied patients' sample, and the major action

Table 5 Comparison Between the Study and Control Groups of Patients Regards to the Frequency of Vital Signs Measurement

\begin{tabular}{|l|c|c|c|}
\hline Frequency of Vital Signs & Control Group & Study Group & p-value \\
\hline Total number of patients & 190 & 174 & $<0.001$ \\
Mean+ SD & $9 \pm 6$ & $15 \pm 13$ & \\
\hline
\end{tabular}

Note: Statistically significant at $\mathrm{p}$-value $<0.05$. 
Table 6 Comparison Between the Study and Control Groups of Patients in Relation to NEWS Escalation Categories

\begin{tabular}{|l|c|c|c|c|}
\hline Escalation Categories (Number \%) & Control Group & Study Group & Crude OR (95\% CI) & p-value \\
\hline Total number of patients & 190 & 174 & & \\
No escalation & $184(96.9 \%)$ & $128(73.6 \%)$ & $0.75(0.18,3.0)$ & $<0.01$ \\
Close observation & $5(2.6 \%)$ & $31(17.8 \%)$ & $6.8(1.6,27.0)$ & $<0.001$ \\
Medication management & $1(0.5 \%)$ & $10(5.7 \%)$ & $11.4(2.7,46.0)$ & $<0.01$ \\
Transferred to ICU & $0(0 \%)$ & $4(2.3 \%)$ & $0.02(0.004,0.08)$ & $<0.01$ \\
Transferred to OR & $0(0 \%)$ & $1(0.6 \%)$ & $0.006(0.001,0.02)$ & $>0.05$ \\
\hline
\end{tabular}

Note: Statistically significant at $p$-value $<0.05$.

plan is taken by the physician based on their medical evaluation upon nurse request was close observation order $(5 / 190$ [2.6\%] vs 31/174 [17.8\%], p $=<0.001)$ of control and study groups, respectively.

It is apparent from Table 7 that reveals that there was no effect of gender or age on outcomes, so the initial differences between groups are not effective in the analysis. Also, there was a significant effect of the intervention in three out of six outcomes, indicating the difference in these outcomes between study and control groups.

\section{Discussion}

\section{Vital Signs Measurement}

Effective observation is the first critical step in identifying the deteriorating patient and effectively managing their care. ${ }^{23}$ Vital signs play an essential role in hospital wards to determine patients at risk of deterioration. ${ }^{24,25}$ Abnormalities in vital sign measurements may indicate a lack of tissue oxygenation, leading to multi-organ dysfunction, and an increase in-hospital mortality rate. ${ }^{26}$ Early detections of these abnormal vital signs could lead to proper and timely treatment, less organ dysfunction, and a lower risk of death. ${ }^{27,28}$ Early detection cannot occur unless vital signs are monitored and documented on a regular basis. ${ }^{3}$ An obvious strategy that is frequently overlooked. ${ }^{29}$ However, this may have happened due to the implementation of rapid response systems. ${ }^{30}$

The study findings revealed that there were highly significant statistical differences between the control and study groups concerning the frequency of vital signs measurement. As during the control group, the number of times to measure the vital signs was not standard. In another term, the staff could measure vital signs twice or only once per shift. Also, they commonly measure only two parameters. In comparison, during the study group, they measured all vital signs parameters as per NEWS guidelines, so the mean number of vital signs measured increased significantly in the study group. This was also seen in other studies. ${ }^{3,6}$

Although the respiratory rate is one of the first vital signs to deviate when a patient is deteriorating, and the respiratory rate's deviation is related to severe deterioration within 48 $\mathrm{hr}^{31}$ the respiratory rate counting was not part of daily nursing practice before implementing the NEWS intervention. A possible explanation for increasing the frequency of all vital signs measurement includes the education program, and the nurses became more aware of the importance of vital sign measurements. This is in line with similar findings ${ }^{32,33}$ as well as the agreement from hospital administration to apply this study mandating the staff to calculate a total

Table 7 Summary of Logistic Regression Analysis for the Effect of the Intervention, Gender, and Age on Each Outcome

\begin{tabular}{|l|c|c|c|}
\hline \multirow{2}{*}{ Outcomes } & Intervention Effect & Gender & Age \\
\cline { 2 - 4 } & \multicolumn{3}{|c|}{ p-value } \\
\hline Cardio Pulmonary Arrest & 0.03 & 0.52 & 0.06 \\
Unplanned ICU Admission & 0.09 & 0.83 & 0.84 \\
Emergency Surgery & 0.99 & 0.31 & 0.09 \\
Unexpected Death & 0.23 & 0.86 & 0.9 \\
Total Acute kidney Injury & 0.03 & 0.23 & 0.26 \\
Escalation Plan & $<0.001$ & 0.96 & 0.08 \\
\hline
\end{tabular}

Note: Statistically significant at $\mathrm{p}$-value $<0.05$. 
NEWS every time a set of observations were performed. Hence, the focus on vital signs documentation to get a total score might help the staff nurses to communicate more succinctly with the health care team.

\section{Medical Review and Escalation Plan}

The monitoring of a clinically deteriorating patient must be associated with an appropriate treatment to improve care, which can only occur following a proper medical review triggered by a meaningful communication by the bedside nurse to the physician. During the intervention period, there was also a significant increase in the number of medical reviews for clinically deteriorated patients, which in turn showed highly significant statistical differences between the study and control group in relation to escalation categories, and the major action plan taken by the physician based on their medical review was close observation order. Also, it allowed for the referral of the patients to ICU and operating room. It was evident that the NEWS is crucial in this process, having the possibility to alert the adequate MET and assist the health care staff in initiating and performing immediate resuscitation attempts while waiting for qualified help. This finding matched with similar studies. ${ }^{3,34}$

The improvement in the instances of patients receiving medical reviews may have occurred by introducing a unified structure for the staff nurses, which not only guide them to request a medical review through reporting of physiological deterioration but also being provided with objective method and structure for communicating the clinical instability. ${ }^{15,35}$ However, escalating care for a deteriorating patient is vital to ensure that treatment is promptly provided. The response was sometimes delayed due to physicians' workloads. This lack of commitment from medical staff to respond to deteriorating patients is consistent with other research studies. ${ }^{36,37}$

\section{Patients Related Outcomes}

Cardiopulmonary Arrest, Unplanned ICU Admission and Emergency Surgery

Detecting patients whose condition is deteriorating is of crucial importance in all areas of practice. Our study showed that introducing the national early warning system led to a significant reduction in the number of cardiopulmonary arrests, unplanned ICU admission, and emergency surgery. These findings have potential consequences for efforts aimed at improving the quality of care for clinically deteriorating patients.
Possible reasons may interpret the significant reduction of these adverse events as the early recognition, response, and treatment of deteriorating patients within a timely manner is a fundamental part of reducing adverse events and improving patient outcomes. This can be explained by increasing the number of medical reviews. This point of view is consistent with Subbe \& Welch $^{38}$ who conducted a study entitled "Failure to rescue: using rapid response systems to improve the care of the deteriorating patient in hospital", and the result revealed that reliable recording of vital signs, recognition of abnormalities, communication of concerns and a timely response could dramatically reduce adverse events and improve outcomes. A similar finding was seen. ${ }^{3,17,39,40}$

To our knowledge, the majority of studies do not specifically address the effect of early warning scoring systems on emergency surgery. Our study showed a highly significant reduction in the numbers of emergency surgery $(12 / 190[6.3 \%]$ vs $0 / 174[0 \%], p=0.001)$. A possible reason for the NEWS risk classification is that it offers a simple way to identify deteriorating patients through a standardized observation system that guides the healthcare staff to prioritize amongst patients. This point of view is in line with a study done by Ludikhuize et al, ${ }^{18}$ who carried out research entitled "Identification of deteriorating patients on general wards; measurement of vital parameters and potential effectiveness of the Modified Early Warning Score," it was a retrospective study and analyzed severe adverse events (SAEs) including emergency surgery. And the study revealed that more than $80 \%$ of patients with serious adverse events, such as emergency surgery, could have been detected earlier using the early warning score, based on the deterioration of vital signs that were noticed at least once in the 48 hours before the adverse event occurrence.

The researcher examined the comparison between both groups of patients as regards all survival rates. It revealed no significant statistical differences between the control and study groups related to all survival rates. However, there was a reduction in the number of all hospital deaths $(12 / 190$ [6.3\%] vs 4/174 [2.3\%], $p=0.062)$. This finding is supported by Farenden et al, ${ }^{41}$ and the result revealed no statistically significant differences between the overall hospital mortality and the outcomes before or after NEWS implementation. On the contrary, this finding is not agreed with Moon et $\mathrm{al}^{42}$ study that revealed a significant reduction in the in-hospital mortality after introducing the early warning scoring system $(52 \%$ vs $42 \% ; \mathrm{p}=0.05)$. 


\section{Acute Kidney Injury}

The study findings revealed a significant reduction in total acute kidney injury among the study group compared to the control group. A possible reason for this significant reduction could be increased medical reviews for unstable patients, enabling the physician for early detection and proper management. This finding contradicts the study entitled "Outcomes in patients with acute kidney injury reviewed by critical care outreach: What is the role of the National Early Warning Score?" conducted by Potter et al, ${ }^{43}$ and the result revealed that the NEWS was a poor predictor of mortality in the study, suggesting that NEWS has little role in the escalation of the patient with AKI. Also, Mitchell et al ${ }^{3}$ study added that the number of acute kidney injury decreased but without significance $(1 / 1157$ vs $0 / 985, \mathrm{p}=1.00)$.

In light of little researchers studying the effect of NEWS on acute kidney injury (AKI), the researcher viewed the current finding as the patients with AKI are frequently poorly identified and managed. However, AKI could be preventable and treatable. This point of view is in line with Hulse \& Davies $^{44}$ revealed that acute kidney injury (AKI) is common and often avoidable. Nurses should recognize it and respond when it occurs through prevention or early detection, which can help reduce morbidity and mortality associated with AKI, improving patients' quality of life.

\section{Limitation}

The limitation of this study are as follows: First, investigation findings are less amenable to generalization because the sample was selected from one geographical area in Egypt, with a short review period with the post-NEWS data collected only three months after the introduction of the intervention. Also, the majority of the included sample were surgical patients. Second, the scope of the study is also limited. It was restricted to those patients who agreed to participate in the study, not the whole admitted population. Third, not all ward patients requiring ICU admission according to the NEWS score were transferred to ICU immediately. Nursing staff responsible for the patient may have been the first to be contacted, in which some of the ICU admissions were delayed when the ICU Team was unavailable. Finally, a possible limitation due to the use of a control group may be risk bias due to other concomitant changes.

\section{Conclusion}

Based on the findings of the current study, it can be concluded that the implementation of NEWS within an emergency hospital is effective in the identification and initiation of early intervention for patients who present with or develop a critical illness. It also has a greater ability to decrease the numbers of patients at risk of cardiac arrest, unplanned ICU admission, emergency surgery, acute kidney injury, as well as an increase in the number of patients receiving medical reviews following clinical deterioration.

\section{Acknowledgments}

I would like to acknowledge the contribution of all the participants who kindly agreed to take part in the study. They generously gave their time and attention to conduct this study. This study would have been impossible without their generosity.

\section{Disclosure}

The author reports no conflicts of interest in this work.

\section{References}

1. Massey D, Chaboyer W, Anderson V. What factors influence ward nurses' recognition of and response to patient deterioration? An integrative review of the literature. Nurs Open. 2016;4(1):6-23. doi:10.1002/nop2.53

2. Padilla RM, Mayo AM. Clinical deterioration: a concept analysis. J Clin Nurs. 2018;27(7-8):1360-1368. doi:10.1111/jocn.14238

3. Mitchell IA, McKay H, Van Leuvan C, et al. A prospective controlled trial of the effect of a multi-faceted intervention on early recognition and intervention in deteriorating hospital patients. Resuscitation. 2010;81(6):658-666. doi:10.1016/j.resuscitation.2010.03.001

4. Connell CJ, Endacott R, Cooper S. The prevalence and management of deteriorating patients in an Australian emergency department. Australas Emerg Care. 2020;S2588-994X(20)30067-1. doi:10.1016/ j.auec.2020.07.008

5. Mok WQ, Wang W, Liaw SY. Vital signs monitoring to detect patient deterioration: an integrative literature review. Int $J$ Nurs Pract. 2015;21(Suppl 2):91-98. doi:10.1111/ijn.12329

6. Hammond NE, Spooner AJ, Barnett AG, Corley A, Brown P, Fraser JF. The effect of implementing a modified early warning scoring (MEWS) system on the adequacy of vital sign documentation. Aust Crit Care. 2013;26(1):18-22. doi:10.1016/j.aucc.2012.05.001

7. Stevenson JE, Israelsson J, Nilsson GC, Petersson GI, Bath PA. Recording signs of deterioration in acute patients: the documentation of vital signs within electronic health records in patients who suffered in-hospital cardiac arrest. Health Informatics J. 2016;22(1):21-33. doi: $10.1177 / 1460458214530136$

8. Santos MD, Clifton DA, Tarassenko L. Performance of early warning scoring systems to detect patient deterioration in the emergency department. In: Gibbons J, MacCaull W, editors. Foundations of Health Information Engineering and Systems. FHIES 2013. Lecture Notes in Computer Science. Vol. 8315. Berlin, Heidelberg: Springer; 2014. doi:10.1007/978-3-642-53956-5_11

9. Royal College of Physicians. National early warning score (NEWS) 2. Standardizing the assessment of acute-illness severity in the NHS; 2017. Available from: https://www.rcplondon.ac.uk. Accessed March 24, 2021.

10. Royal College of Physicians. National Early Warning Score (NEWS): standardizing the assessment of acute illness severity in the NHS. Report of a working party. RCP; 2012. Available from: https://www. rcplondon.ac.uk. Accessed December 3, 2021. 
11. World Health Organization. Clinical management of severe acute respiratory infection when COVID-19 is suspected. Interim guidance; 2020. Available from: https://www.who.int/publications/i/ item/WHO-2019-nCoV-clinical-2021-1. Accessed April 23, 2021.

12. Royal College of Physicians. NEWS2 and deterioration in COVID; 2021. Available from: https://www.rcplondon.ac.uk/news/news2-anddeterioration-covid-19. Accessed April 23, 2021.

13. Rigoni M, Torri E, Nollo G, Delle Donne L, Cozzio S. NEWS2 is a valuable tool for appropriate clinical management of COVID-19 patients. Eur $J$ Intern Med. 2021;85:118-120. doi:10.1016/j. ejim.2020.11.020

14. Spagnolli W, Rigoni M, Torri E, Cozzio S, Vettorato E, Nollo G. Application of the National Early Warning Score (NEWS) as a stratification tool on admission in an Italian acute medical ward: a perspective study. Int J Clin Pract. 2017;71(3-4):e12934. doi:10.1111/ ijcp. 12934

15. Andrews T, Waterman H. Packaging: a grounded theory of how to report physiological deterioration effectively. $J$ Adv Nurs. 2005;52 (5):473-481. doi:10.1111/j.1365-2648.2005.03615.x

16. Mehta RL, Kellum JA, Shah SV, et al.; Acute Kidney Injury Network. Acute kidney injury network: report of an initiative to improve outcomes in acute kidney injury. Crit Care. 2007;11(2): R31. doi:10.1186/cc5713

17. Spångfors M, Arvidsson L, Karlsson V, Samuelson K. The National Early Warning Score: translation, testing and prediction in a Swedish setting. Intensive Crit Care Nurs. 2016;37:62-67. doi:10.1016/j. iccn.2016.05.007

18. Ludikhuize J, Smorenburg SM, de Rooij SE, de Jonge E. Identification of deteriorating patients on general wards; measurement of vital parameters and potential effectiveness of the Modified Early Warning Score. J Crit Care. 2012;27(4):424.e7-13. doi:10.1016/j.jcrc.2012.01.003

19. Cretikos M, Parr M, Hillman K, et al. Guidelines for the uniform reporting of data for Medical Emergency Teams. Resuscitation. 2006;68(1):11-25. doi:10.1016/j.resuscitation.2005.06.009

20. Anathhanam S, Lewington AJ. Acute kidney injury. $J R$ Coll Physicians Edinb. 2013;43(4):323-8;quiz 329. doi:10.4997/ JRCPE.2013.412

21. Lopes JA, Jorge S. The RIFLE and AKIN classifications for acute kidney injury: a critical and comprehensive review. Clin Kidney J. 2013;6(1):8-14. doi:10.1093/ckj/sfs 160

22. Ostermann M, Joannidis M. Acute kidney injury 2016: diagnosis and diagnostic workup. Crit Care. 2016;20(1):299. doi:10.1186/s13054016-1478-z

23. Odell M, Victor C, Oliver D. Nurses' role in detecting deterioration in ward patients: systematic literature review. $J A d v$ Nurs. 2009;65 (10):1992-2006. doi:10.1111/j.1365-2648.2009.05109.x

24. Mok W, Wang W, Cooper S, Ang EN, Liaw SY. Attitudes towards vital signs monitoring in the detection of clinical deterioration: scale development and survey of ward nurses. Int J Qual Health Care. 2015;27(3):207-213. doi:10.1093/intqhe/mzv019

25. Cardona-Morrell M, Prgomet M, Lake R, et al. Vital signs monitoring and nurse-patient interaction: a qualitative observational study of hospital practice. Int J Nurs Stud. 2016;56:9-16. doi:10.1016/j. ijnurstu.2015.12.007

26. Hogan H, Healey F, Neale G, Thomson R, Vincent C, Black N. Preventable deaths due to problems in care in English acute hospitals: a retrospective case record review study. BMJ Qual Saf. 2012;21 (9):737-745. doi:10.1136/bmjqs-2011-001159

27. Mora JC, Schneider A, Robbins R, et al. Epidemiology of early rapid response team activation after emergency department admission. Australas Emerg Nurs J. 2016;19(1):54-61. doi:10.1016/j. aenj.2015.05.001
28. Subbe CP, Duller B, Bellomo R. Effect of an automated notification system for deteriorating ward patients on clinical outcomes. Crit Care. 2017;21(1):52. doi:10.1186/s13054-017-1635-z

29. Hillman K, Chen J, Cretikos M, et al.; MERIT study investigators. Introduction of the medical emergency team (MET) system: a cluster-randomized controlled trial. Lancet. 2005;365 (9477):2091-2097. doi:10.1016/S0140-6736(05)66733-5

30. Chen J, Hillman K, Bellomo R, Flabouris A, Finfer S, Cretikos M; MERIT Study Investigators for the Simpson Centre; ANZICS Clinical Trials Group. The impact of introducing medical emergency team system on the documentations of vital signs. Resuscitation. 2009;80(1):35-43. doi:10.1016/j.resuscitation.2008.10.009

31. Bunkenborg G, Poulsen I, Samuelson K, Ladelund S, Akeson J. Bedside vital parameters that indicate early deterioration. Int J Health Care Qual Assur. 2019;32(1):262-272. doi:10.1108/ IJHCQA-10-2017-0206

32. Bliss M, Aitken LM. Does simulation enhance nurses' ability to assess deteriorating patients? Nurse Educ Pract. 2018;20-26. doi:10.1016/j.nepr.2017.09.009.

33. Jensen JK, Skår R, Tveit B. Introducing the national early warning score - a qualitative study of hospital nurses' perceptions and reactions. Nurs Open. 2019;6(3):1067-1075. doi:10.1002/nop2.291

34. Leuvan CH, Mitchell I. Missed opportunities? An observational study of vital sign measurements. Crit Care Resusc. 2008;10(2):111-115.

35. Bunkenborg G, Samuelson K, Poulsen I, Ladelund S, Åkeson J. Lower incidence of unexpected in-hospital death after interprofessional implementation of a bedside track-and-trigger system. Resuscitation. 2014;85(3):424-430. doi:10.1016/j. resuscitation.2013.11.023

36. Fox A, Elliott N. Early warning scores: a sign of deterioration in patients and systems. Nurs Manag (Harrow). 2015;22(1):26-31. doi:10.7748/nm.22.1.26.e1337

37. Foley C, Dowling M. How do nurses use the early warning score in their practice? A case study from an acute medical unit. J Clin Nurs. 2019;28(7-8):1183-1192. doi:10.1111/jocn. 14713

38. Subbe CP, Welch JR. Failure to rescue: using rapid response systems to improve care of the deteriorating patient in hospital. Clin Risk. 2013;19(1):6-11. doi:10.1177/1356262213486451

39. Smith GB, Prytherch DR, Meredith P, Schmidt PE, Featherstone PI. The ability of the National Early Warning Score (NEWS) to discriminate patients at risk of early cardiac arrest, unanticipated intensive care unit admission, and death. Resuscitation. 2013;84(4):465-470. doi:10.1016/j.resuscitation.2012.12.016

40. Yuan WC, Tao C, Dan ZD, Yi SC, Jing W, Jian Q. The significance of National Early Warning Score for predicting prognosis and evaluating conditions of patients in resuscitation room. Hong Kong J Emerg Med. 2018;25(6):324-330. doi:10.1177/1024907918775879

41. Farenden S, Gamble D, Welch J. Impact of implementation of the National Early Warning Score on patients and staff. Br J Hosp Med (Lond). 2017;78(3):132-136. doi:10.12968/hmed.2017.78.3.132

42. Moon A, Cosgrove JF, Lea D, Fairs A, Cressey DM. An eight year audit before and after the introduction of modified early warning score (MEWS) charts, of patients admitted to a tertiary referral intensive care unit after CPR. Resuscitation. 2011;82(2):150-154. doi:10.1016/j.resuscitation.2010.09.480

43. Potter DA, Wroe N, Redhead H, Lewington AJ. Outcomes in patients with acute kidney injury reviewed by critical care outreach: what is the role of the National Early Warning Score? J Intensive Care Soc. 2017;18(4):300-309. doi:10.1177/1751143717715968

44. Hulse C, Davies A. Acute kidney injury: prevention and recognition. Nurs Times. 2015;111(30-31):12-15. 


\section{Publish your work in this journal}

The Journal of Multidisciplinary Healthcare is an international, peerreviewed open-access journal that aims to represent and publish research in healthcare areas delivered by practitioners of different disciplines. This includes studies and reviews conducted by multidisciplinary teams as well as research which evaluates the results or conduct of such teams or healthcare processes in general. The journal covers a very wide range of areas and welcomes submissions from practitioners at all levels, from all over the world. The manuscrip management system is completely online and includes a very quick and fair peer-review system. Visit http://www.dovepress.com/testimonials. php to read real quotes from published authors. 